\title{
Anti-CXCL4 monoclonal antibody accelerates telogen to anagen transition and attenuates apoptosis of the hair follicle in mice
}

\author{
WEN GUAN ${ }^{1,2}$, XIAOLAN YU ${ }^{1},{\text { JINGJING } \text { LI }^{3} \text {, } \text { QING DENG }^{1} \text {, YANG ZHANG }}^{3}$, JING GAO $^{3}$, \\ PENG XIA ${ }^{1}$, YUNSHENG YUAN ${ }^{3}$, JIN GAO $^{3}$, LIANG ZHOU ${ }^{1}$, WEI HAN ${ }^{3}$ and YAN YU ${ }^{1}$ \\ ${ }^{1}$ Shanghai Municipality Key Laboratory of Veterinary Biotechnology, \\ Department of Animal Sciences, School of Agriculture and Biology, Shanghai Jiaotong University, Shanghai 200240; \\ ${ }^{2}$ Department of Biochemistry, Zhongshan School of Medicine, Sun Yat-Sen University, Guangzhou, Guangdong 510080; \\ ${ }^{3}$ Laboratory of Regeneromics, School of Pharmacy, Shanghai Jiaotong University, Shanghai 200240, P.R. China
}

Received September 16, 2016; Accepted April 10, 2017

DOI: 10.3892/etm.2017.4578

\begin{abstract}
Although hair loss or alopecia is a common disease, its exact mechanisms are not yet well understood. The present study investigated the hypothesis that the homeostatic regulation of genes during hair regeneration may participate in hair loss, based on the cyclicity of hair growth. A cluster of such genes was identified by an expression gene-array from the dorsal skin in a depilated mouse model, and CXCL4 was identified as a significantly regulated gene during the hair regeneration process. To elucidate the function of CXCL4 in hair growth, CXCL4 activity was blocked by the administration of an anti-CXCL4 monoclonal antibody (mAb). Histomorphometric analysis indicated that anti-CXCL4 $\mathrm{mAb}$ induced an earlier anagen phase and delayed hair follicle regression, in contrast with that in the control group. Moreover, CXCL4 mAb upregulated the transcription levels of several hair growth-related genes, including Lef1, Wnt10b, Bmp4 and Bmp2. In addition, CXCL4 $\mathrm{mAb}$ increased the levels of the proliferation-related protein PCNA and Bcl-2 during the anagen phase, while it reduced the expression of pro-apoptotic protein Bax and cleaved caspase-3 during the catagen phase. These findings reveal that CXCL4 plays an important role in hair growth, and that blockade of CXCL4 activity promotes hair growth.
\end{abstract}

Correspondence to: Professor Yan Yu, Shanghai Municipality Key Laboratory of Veterinary Biotechnology, Department of Animal Sciences, School of Agriculture and Biology, Shanghai Jiaotong University, 800 Dongchuan Road, Shanghai 200240, P.R. China

E-mail: yanyu@sjtu.edu.cn

Professor Wei Han, Laboratory of Regeneromics, School of Pharmacy, Shanghai Jiaotong University, 800 Dongchuan Road, Shanghai 200240, P.R. China

E-mail: weihan@sjtu.edu.cn

Key words: CXCL4, hair follicle, hair cycle, anagen, catagen

\section{Introduction}

Hair is considered to be an accessory structure of the integumentary system, along with sebaceous glands, sweat glands and nails. Hair growth is cyclic, involving a growth (anagen) phase, an apoptosis-mediated regression (catagen) phase and a resting (telogen) phase $(1,2)$. Hair loss or alopecia is becoming more prevalent worldwide (3). Although hair loss is generally not life-threatening, it has been shown to have a substantial negative impact on social interactions and psychological well-being (4). To date, most marketed anti-hair-loss products are ineffective. Finasteride and minoxidil are the only two products approved for hair loss by the US Food and Drug Administration. However, their use is limited owing to unpredictable efficacy and undesirable side effects (5). Therefore, it is urgently necessary to develop novel pharmacological treatments.

Various cytokines and growth factors contribute to the regulation of hair morphogenesis and the hair growth cycle. Chemokine (C-X-C motif) ligand 4 (CXCL4; also known as platelet factor-4) is a member of the CXC chemokine family of small (8-10 kDa) proteins (6). CXCL4 was first described by Deutsch et al in 1955 and was demonstrated to be a platelet protein with anti-heparin activity (7). CXCL4 is a $7.8-\mathrm{kDa}$ protein comprising 70 amino acids, that is synthesized in megakaryocytes, expressed in other cells, and stored in $\alpha$-granules (8). CXCL4 has been reported to have numerous biological effects, including immunization, apoptosis, cell differentiation, survival, proliferation and tissue repair effects (9). CXCL4 inhibits the spontaneous apoptosis of monocytes and mediates their differentiation into a specific subtype of macrophages $(9,10)$. It also promotes the survival of hematopoietic stem cells and progenitor cells (11). Moreover, CXCL4 has been reported to have an antiproliferative effect on endothelial cells and fibroblasts, in addition to anti-angiogenic activity (12-15). In addition, CXCL4 participates in mediation of the cell recruitment and activation necessary for inflammation and the repair of tissue damage (6). However, to the best of our knowledge, the role of CXCL4 in the hair growth cycle has not yet been reported. 
Hair shafts are made by the hair follicle, which undergoes self-renewal when hairs are damaged. The present study is based on the proposal that homeostatically regulated gene expression during the hair cycle is essential for hair growth. In order to identify those genes, a genome-wide gene expression array using a depilation-induced hair growth mouse model was performed.

The aim of the present study was to determine whether CXCL4 is an important gene in hair regeneration. Previously, the present authors identified that CXCL4 induced apoptosis of the IEC-6 intestinal epithelial cell line, and that CXCL4 monoclonal antibody $(\mathrm{mAb})$ reduced the apoptosis of the crypt epithelia in a 5-fluorouracil-induced mucositis model (16). Therefore, it was hypothesized that CXCL4 may retard hair growth by exerting an anti-proliferative effect on hair follicle cells, whereas CXCL4 mAb may promote hair growth by stimulating follicular proliferation and delaying the catagen phase. The present study demonstrated that the expression of CXCL4 was downregulated following the transition from the telogen to the anagen phase, and returned to the normal level following the next telogen phase. CXCL4 mAb significantly promoted the initiation of the hair follicle transition from the telogen to the anagen phase through upregulation of hair growth-related genes in vivo. These results indicated that CXCL4 plays an important role in hair growth.

\section{Materials and methods}

Reagents. Antibodies against Bcl-2 (3498), Bax (2772) and the cleaved form of caspase-3 (9664) were purchased from Cell Signaling Technology, Inc. (Danvers, MA, USA). Antibodies against proliferating cell nuclear antigen (PCNA) (sc-25280) and $\beta$-actin (sc-47778) were from Santa Cruz Biotechnology, Inc. (Dallas, TX, USA). Rat immunoglobulin G (IgG) was purchased from Sigma-Aldrich (Merck KGaA, Darmstadt, Germany). CXCL4 mAb was produced in the laboratory, as previously described (16).

Experimental animals. All experiments with mice were conducted in accordance with the Guide for the Care and Use of Laboratory Animals (National Academies Press, Washington, DC, USA, 1996) and were approved by the Animal Research Committee of Shanghai Jiaotong University (Shanghai, China). Male C57BL/6 mice were purchased from Shanghai SLAC Laboratory Animal Co., Ltd. (Shanghai, China). The 6-week-old mice (17-20 g) were allowed to adapt to their new environment for 1 week. Mice were housed in standard animal rooms with food and water available ad libitum under controlled humidity $(50 \pm 15 \%)$ and temperature $\left(22 \pm 2^{\circ} \mathrm{C}\right)$. The room was illuminated by fluorescent lights that were on from 8:00 a.m. to 8:00 p.m.

Microarray analysis. Microarray analysis was conducted by Shanghai Biotechnology Corporation (Shanghai, China). Total RNA was extracted using TRIzol reagent (Thermo Fisher Scientific, Inc., Waltham, MA, USA) from excised C57BL/6 mouse back skin, followed by purification, using RNeasy Mini kit (Qiagen GmBH, Hilden, Germany) and RNase-Free DNase set (Qiagen GmBH). Total RNA was amplified and labeled using a Low Input Quick Amp Labeling kit, One-Color (Agilent Technologies, Inc., Santa Clara, CA, USA). Labeled cRNA was hybridized with Mouse Genome Microarray 4*44K (Agilent Technologies, Inc.) and washed according to the manufacturer's recommendations. Slides were scanned using an Agilent Microarray Scanner (G2565CA; Agilent Technologies, Inc.). The signals were analyzed using Feature Extraction Software 10.7 (Agilent Technologies, Inc.), excluding the data from signals that were recognized as outlier or equal to background. Raw data were normalized by Quantile algorithm, Gene Spring Software 11.0 (Agilent Technologies, Inc.).

Experimental analysis using CXCL4 mAb. Twenty C57BL/6 mice in two randomized groups $(n=10)$ were used for studying the activity of CXCL4 mAb. The anagen phase was induced by depilation on the dorsal skin of the animals at 7 weeks old. This was performed by applying a melted wax/rosin mixture to the dorsal skin and peeling off this mixture after hardening, as previously described (17). All depilated mouse hair follicles were synchronized in the telogen stage. The animals in the test group were subcutaneously injected with CXCL4 mAb (1 $\mathrm{mg} / \mathrm{kg}$ body weight) once a week for three consecutive weeks, and the control group were subcutaneously injected with rat isotype $\mathrm{IgG}$ (1 mg/kg body weight) once a week for three consecutive weeks. The dorsal skin of the mice was observed and photographic images were captured on $0,7,10$ and 18 days after depilation. Three mice from each time point were sacrificed to obtain skin specimens. Briefly, after being euthanized, mice were shaved with mechanical clippers and total, fully-thickness dorsal skin was dissected free. The skin was frozen quickly in liquid nitrogen prior to analysis.

Histological preparation. Dorsal skin of the mice was fixed with $10 \%$ neutral buffered formalin. The tissues were dehydrated through an ascending series of graded ethanol, cleared in xylene, and embedded in paraffin blocks. Subsequently, samples were cut either longitudinally or transversely into $5-\mu \mathrm{m}$ sections. These sections were stained with hematoxylin and eosin (H\&E), and the histological morphology was examined using light microscopy.

Hair follicle counting. Digital photomicrographs were taken from representative areas of slides at a fixed magnification of $\mathrm{x} 40$. All images were cropped in a fixed area with a width of $1,500 \mu \mathrm{m}$. The hair follicles in the deep subcutis were counted manually, as previously described (5).

Reverse transcription-quantitative polymerase chain reaction $(R T-q P C R)$. Total RNA was extracted from the skin using two-step RNA extraction with TRIzol (Invitrogen; Thermo Fisher Scientific, Inc.). Reverse transcription (RT) reaction was performed with RNA $(2 \mu \mathrm{g})$ at $37^{\circ} \mathrm{C}$ for $15 \mathrm{~min}$ and $85^{\circ} \mathrm{C}$ for $5 \mathrm{sec}$ according to the PrimeScript RT reagent kit (Takara Biotechnology Co., Ltd., Dalian, China). The product was amplified in a reaction volume of $20 \mu \mathrm{l}$ containing 9.2 $\mu \mathrm{l}$ RT product, $10 \mu \mathrm{l}$ SYBR Premix Ex Taq II (2x) (Takara Biotechnology Co., Ltd.), and 20 pmol of each primer. PCRs were performed for 40 cycles at $95^{\circ} \mathrm{C}$ for $10 \mathrm{sec}$, $60^{\circ} \mathrm{C}$ for $30 \mathrm{sec}$ and $72^{\circ} \mathrm{C}$ for $30 \mathrm{sec}$ using a $7900 \mathrm{HT}$ Fast Real Time RT-PCR System (Applied Biosystems; Thermo 
Table I. Sequences of forward and reverse primers for reverse transcription-quantitative polymerase chain reaction.

\begin{tabular}{|c|c|c|c|c|}
\hline Genes & Primer & Sequences (5'-3') & Product size (bp) & GenBank accession no. \\
\hline$\beta$-catenin & $\begin{array}{l}\text { Forward } \\
\text { Reverse }\end{array}$ & $\begin{array}{l}\text { GTCAGCTCGTGTCCTGTGAA } \\
\text { GTGCGCTGAGCTTCAGGT }\end{array}$ & 148 & NM_007614.3 \\
\hline Ccnd2 & $\begin{array}{l}\text { Forward } \\
\text { Reverse }\end{array}$ & $\begin{array}{l}\text { CTGAGTCTGGTTGGTGCTGA } \\
\text { ACACCCGAGACCACAGAAAC }\end{array}$ & 239 & NM_009829.3 \\
\hline Lef1 & $\begin{array}{l}\text { Forward } \\
\text { Reverse }\end{array}$ & $\begin{array}{l}\text { GCCACCGATGAGATGATCCC } \\
\text { TTGATGTCGGCTAAGTCGCC }\end{array}$ & 107 & NM_010703 \\
\hline Wnt10b & $\begin{array}{l}\text { Forward } \\
\text { Reverse }\end{array}$ & $\begin{array}{l}\text { TCTTGGATTCCAGGGAGGCT } \\
\text { ACGTTCCATGGCATTTGCAC }\end{array}$ & 120 & NM_011718.2 \\
\hline Wnt5a & $\begin{array}{l}\text { Forward } \\
\text { Reverse }\end{array}$ & $\begin{array}{l}\text { GCTTCGCTTGAATTCCTCGG } \\
\text { ACTTGGAAGACATGGCACCT }\end{array}$ & 143 & NM_009524.3 \\
\hline Bmp4 & $\begin{array}{l}\text { Forward } \\
\text { Reverse }\end{array}$ & $\begin{array}{l}\text { TGAGCCTTTCCAGCAAGTTT } \\
\text { CTTCCCGGTCTCAGGTATCA }\end{array}$ & 180 & NM_007554.2 \\
\hline Bmp2 & $\begin{array}{l}\text { Forward } \\
\text { Reverse }\end{array}$ & $\begin{array}{l}\text { TGGAAGTGGCCCATTTAGAG } \\
\text { TGACGCTTTTCTCGTTTGTG }\end{array}$ & 166 & NM_007553.3 \\
\hline Gli1 & $\begin{array}{l}\text { Forward } \\
\text { Reverse }\end{array}$ & $\begin{array}{l}\text { CCAAGCCAACTTTATGTCAGGG } \\
\text { AGCCCGCTTCTTTGTTAATTTGA }\end{array}$ & 130 & NM_010296 \\
\hline Vcan & $\begin{array}{l}\text { Forward } \\
\text { Reverse }\end{array}$ & $\begin{array}{l}\text { TTTTACCCGAGTTACCAGACTCA } \\
\text { GGAGTAGTTGTTACATCCGTTGC }\end{array}$ & 106 & NM_001081249 \\
\hline$\beta$-actin & $\begin{array}{l}\text { Forward } \\
\text { Reverse }\end{array}$ & $\begin{array}{l}\text { AGCCTTCCTTCTTGGGTATG } \\
\text { GTGTTGGCATAGAGGTCTTTAC }\end{array}$ & 103 & NM_007393 \\
\hline
\end{tabular}

Ccnd2, cyclin D2; Lef1, lymphoid enhancer binding factor 1; Bmp, bone morphogenetic protein; Gli1, GLI-Kruppel family member GLI1; Vcan, versican.

Fisher Scientific, Inc.). Three replicates were performed at each time point. All data were normalized to $\beta$-actin mRNA levels, and the fold change for each mRNA was calculated using the $2^{-\Delta \Delta C q}$ method (18). The primer sequences used are shown in Table I.

Western blot analysis. Skin tissue (100 mg) was homogenized in $1 \mathrm{ml}$ radioimmunoprecipitation assay lysis buffer with protease and phosphatase inhibitors (Beyotime Institute of Biotechnology, Haimen, China). Supernatants were recovered after $12,000 \times \mathrm{g}$ centrifugation at $4^{\circ} \mathrm{C}$ for $10 \mathrm{~min}$, and their protein content was determined using a bicinchoninic acid assay kit (P001; Beyotime Institute of Biotechnology). Proteins $(30 \mu \mathrm{g})$ were separated using 12\% SDS-PAGE gels, and transferred to a polyvinylidene difluoride membrane. Membranes were blocked in 5\% non-fat dry milk for $2 \mathrm{~h}$ at room temperature and incubated with anti-PCNA (1:1,000 dilution, sc-25280, Santa Cruz Biotechnology, Inc.), anti-cleaved caspase-3 (1:1,000 dilution, 9664, Cell Signaling Technology, Inc.), anti-Bcl2 (1:1,000 dilution, 3498, Cell Signaling Technology, Inc.), anti-Bax (1:1,000 dilution, 2772, Cell Signaling Technology, Inc.), and anti- $\beta$-actin (1:1,000 dilution, sc-47778, Santa Cruz Biotechnology, Inc.) primary antibodies overnight at $4^{\circ} \mathrm{C}$ and then with the corresponding HRP-conjugated secondary antibodies (1:5,000, 7044 and 7066, Cell Signaling Technology, Inc.) for $2 \mathrm{~h}$ at room temperature. The membranes were washed and visualized using enhanced chemiluminescence reagents (Thermo Fisher Scientific, Inc.) and analyzed using a gel imaging system (Tanon 3500; Tanon Science and Technology Co., Ltd., Shanghai, China). $\beta$-actin was employed as a loading control. Three replicates were performed for each treatment.

Statistical analysis. All data are presented as the mean \pm standard deviation from three independent experiments. All statistical analysis was performed using SPSS 18.0 (SPSS, Inc., Chicago, IL, USA). Differences between experimental groups were evaluated by Student's t-test. $\mathrm{P}<0.05$ was considered to indicate a statistically significant difference.

\section{Results}

Expression of CXCL4 during the induced mouse hair cycle. In the mouse model used in the present study, a new cycle of hair growth was induced by depilation, as previously described (17). The anagen phase was fully synchronized over the entire area of depilation. At 0 and 26 days, typical telogen morphology, including the entire hair follicle residing in the dermis and lacking melanin, was observed in the hair matrix above the dermal papilla. At 7 and 14 days, enlarged dermal papillae were observed. In addition, elongated hair shafts emerged (Fig. 1A). Gene expression of the total dorsal skin taken from mice at several time points following depilation (7, 14 and 26 days) was analyzed by DNA microarray hybridization and compared with skin samples taken at the telogen phase ( 0 days). The expression of CXCL4 was identified to be decreased during the anagen phase (0-14 days) and returned to the normal level during the telogen phase (26 days; Fig. 1B). The homeostatic regulation of CXCL4 shown in the expression array was confirmed by RT-qPCR (Fig. 1C). The expression 
pattern of CXCL4 in the hair cycle suggests its participation in hair growth following depilation.

CXCL4 mAb promotes hair regeneration. The homeostatic regulation of CXCL4 in the skin strongly suggests its role in hair growth. A strategy of using a neutralizing $\mathrm{mAb}$ to block CXCL4 was used to evaluate the role of CXCL4 in hair growth. Adult C57BL/6 mice are useful for testing hair growth-promoting reagents, as their truncal pigmentation is dependent on their follicle melanocytes, which produce pigment only during anagen (19). It has been reported that the skin color of mice is pink in the telogen phase, and gradually becomes grey and black along with anagen initiation (20). Thus, black pigmentation is taken as evidence of the transition of hair follicles from the telogen to the anagen phase.

To evaluate the hair growth activity of CXCL4 mAb, the depilated mice were treated with CXCL4 mAb once a week, for 3 weeks. Each day, the degree of hair growth was evaluated by observing the skin color. Darker skin was observed in the CXCL4 mAb-treated group at 7 and 10 days, compared with that of the control mice treated with isotype $\operatorname{IgG}$ (Fig. 2). These results indicate that $\mathrm{CXCL} 4 \mathrm{mAb}$ significantly stimulated hair growth.

Effects of CXCL4 $\mathrm{mAb}$ on the development and structure of mouse hair follicles. An increase in the number of hair follicles has been considered as an indicator of the transition of hair growth from the telogen to the anagen phase. The anagen phase is associated with an increase in the number of hair follicles that lie in the deep subcutis compared with that of the telogen phase, in which hair follicles lie in the dermis only (20). The effect of CXCL4 mAb on hair follicle density was further assessed via histological analysis of mouse skin samples following staining with H\&E (Fig. 3A-F). CXCL4 $\mathrm{mAb}$ significantly increased the number of hair follicles in mice, compared with that in the control group at 10 and 18 days $(\mathrm{P}<0.05$; Fig. $3 \mathrm{G})$.

In accordance with the classification of hair cycle stages in mice (1), hair follicles at 18 days following depilation are in the catagen phase. Longitudinal sections of dorsal skins are shown in Fig. 3H-K. It was observed that regression of the hair follicles pulled the dermal papillae upward into the dermis in the control group (Fig. 3I). By contrast, almost all of the hair follicles in the CXCL4 mAb-treated group continued to reside in the subcutis (Fig. $3 \mathrm{~K}$ ). These observations indicate that blockade of CXCL4 induced an earlier anagen phase and prolonged the mature anagen phase, compared with that in the control group.

CXCL4 mAb upregulates Wnt- and bone morphogenetic protein (Bmp)-dependent signaling. Previous studies demonstrated that transient activation of Wnt-dependent $\beta$-catenin signaling in cutaneous keratinocytes is sufficient to trigger the active growth phase of the hair cycle in mice $(21,22)$. Therefore, total RNA was isolated from C57BL/6 skin samples at 10 days after depilation and expression levels of Wnt pathway genes were examined using RT-qPCR. Increased mRNA levels of $\beta$-catenin (2.0-fold), cyclin D2 (Ccnd2; 1.4-fold), lymphoid enhancer binding factor 1 (Lef1; 2.8-fold), Wnt10b (2.3-fold) and Wnt5a (1.5-fold) were observed in the mice treated with CXCL4 mAb compared with the control mice (Fig. 4A).

Previous studies have revealed that the Bmp pathway may provide a molecular target for anagen induction and hair growth in mice and humans (23), and that Wntla-conditioned medium upregulates hair-induction related genes (24). Similar to the aforementioned RT-qPCR experiments, increased mRNA levels of Bmp4 (2.2-fold), Bmp2 (2.7-fold), GLI-Kruppel family member GLI1 (Gli1; 2.5-fold) and versican (Vcan; 1.2-fold) were found in the mice treated with CXCL4 mAb in comparison with the control mice (Fig. 4B).

Effects of CXCL4 mAb on proliferation- and apoptosis-associated proteins. Whole-tissue lysates of dorsal skin tissues were utilized to investigate the effects of CXCL4 mAb on proliferation- and apoptosis-associated factors in hair growth and regression. At 10 days, CXCL4 mAb was observed to significantly increase Bcl-2 (1.5-fold) and PCNA (1.8-fold) expression during the anagen phase, compared with that in the control group (Fig. 5A and B). In addition, at 18 days, CXCL4 $\mathrm{mAb}$ treatment significantly reduced the protein expression of two apoptosis-associated factors, Bax (1.6-fold) and cleaved caspase-3 (1.9-fold), compared with that in the control group, indicating that CXCL4 $\mathrm{mAb}$ prevented hair follicles from undergoing apoptosis (Fig. 5C and D). These results indicate that CXCL4 $\mathrm{mAb}$ promoted hair regrowth and delayed hair regression.

\section{Discussion}

In the present study, a novel role for CXCL4 $\mathrm{mAb}$ in the promotion of hair growth in mice following depilation was uncovered. To the best of our knowledge, the present study is the first to report an association between CXCL4 and hair growth. It demonstrated that dorsal skin expressed low basal level of CXCL4 mRNA during the anagen phase of hair growth, and the expression level returned to normal following the next telogen phase. Furthermore, the present study demonstrated that CXCL4 was involved in the control of hair growth, and that neutralization of CXCL4 using a mAb significantly promoted hair growth in a depilated mouse model.

Previous studies have shown that CXCL4 inhibits endothelial cell proliferation and interferes with the cell cycle $(12,25)$. It has been reported that CXCL4-stimulated human monocytes induce the apoptosis of endothelial cells via the release of oxygen radicals (26). This is consistent with our previous study, in which recombinant human CXCL4 was observed to induce the apoptosis of intestinal epithelial cells, CXCL4 mAb was demonstrated to protect mouse intestine from chemotoxicity (16). To the best of our knowledge, there are no previous reports concerning the effect of CXCL4 or anti-CXCL4 antibody on hair growth. In the present study, it was identified that the expression of CXCL4 was downregulated during the anagen phase and returned to the basal level at the telogen phase. The effect of CXCL4 mAb on hair growth was also investigated in the present study. The results suggest that CXCL4 mAb induces hair growth by promoting telogen to anagen transition of hair follicles, and suppresses apoptosis of hair follicles during the catagen phase in mice. 
A

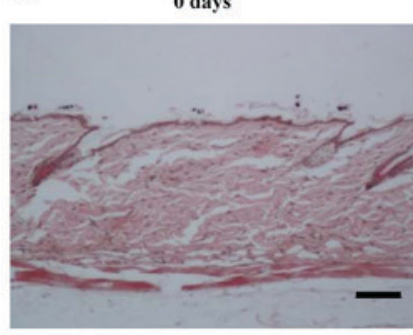

B

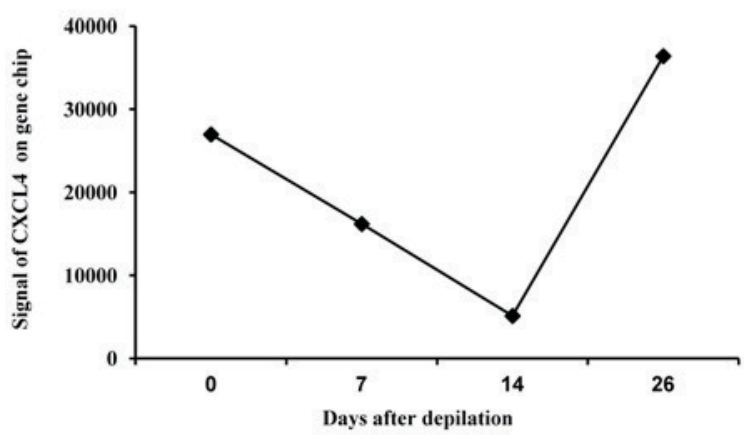

7 days

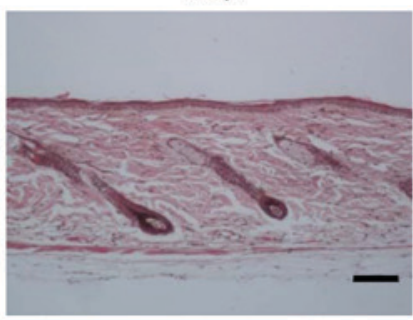

C
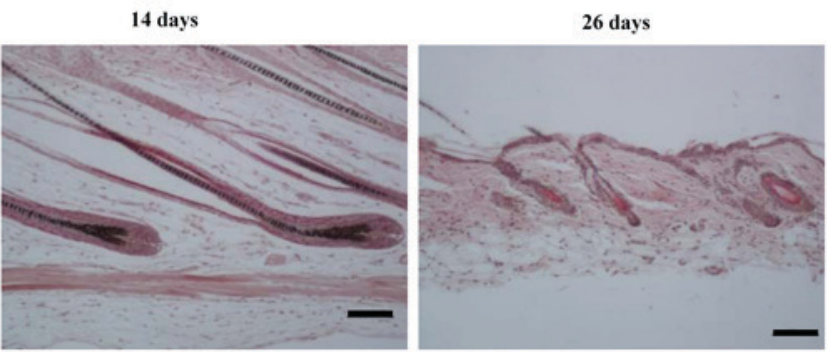

26 days

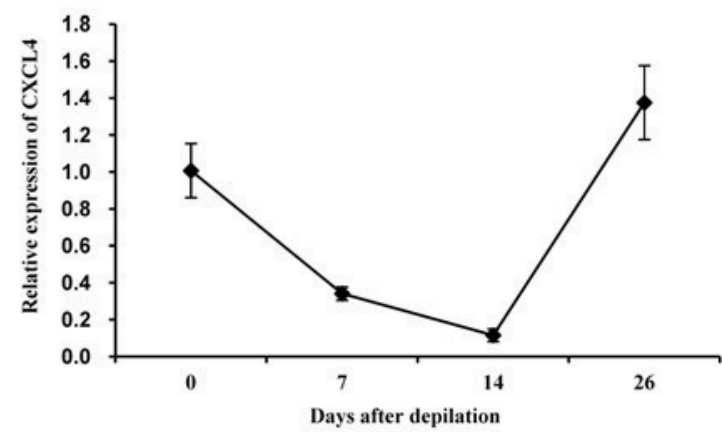

Figure 1. Expression profile of CXCL4 at different phases of the hair growth cycle. The anagen was induced in the dorsal skin of C57BL/6 mice by depilation. (A) Changes in hair follicle morphology at 7, 14 and 26 days after depilation; 0 days indicates hair in the telogen phase, prior to depilation (n=3). Scale bar, $100 \mu \mathrm{m}$. (B) Expression level of CXCL4 mRNA at different phases of the hair growth cycle as detected by microarray (n=3). (C) Expression level of CXCL4 mRNA at different phases of the hair growth cycle as determined by reverse transcription-quantitative polymerase chain reaction ( $\mathrm{n}=3$ ). $\mathrm{CXCL} 4$, chemokine (C-X-C motif) ligand 4.

Hair cycling between the telogen and anagen phases is precisely regulated by dermal papilla (DP) cells. The Wnt pathway is an important signaling pathway that activates the hair induction ability of DP cells (27). $\beta$-catenin and Wnt5a expression are moderate in the early anagen phase, high in the middle anagen phase, and weak in the catagen and telogen phases $(21,28)$. Adenovirus-mediated ectopic expression of Wnt10b was able to activate precocious anagen entry in a previous mouse model (29). Lef1 is an essential regulatory protein in the Wnt-signaling pathway that controls cell growth and differentiation (30). In the stem cell niche, Wnt signaling and $\beta$-catenin stabilization are considered to activate the Lef1/T-cell factor complex, which binds to its target genes and promotes stem cell activation and proliferation; these constitute critical steps required to support hair growth (31). Moreover, the Bmp signal transduction pathway also plays an important role in anagen induction (23). In the present study, elevated mRNA levels of Wnt- $\beta$-catenin signaling molecules were detected, including $\beta$-catenin, Ccnd2, Lef1, Wnt10b and Wnt5a at 10 days after depilation in mice (Fig. 4A). Furthermore, increased levels of Bmp4, Bmp2, Gli1 and Vcan were also found in mice treated with CXCL4 mAb (Fig. 4B). These findings are consistent with previous studies in which the expression levels of hair induction-related genes were upregulated in hair regrowth $(24,32)$. The data demonstrate that CXCL4 mAb activates the Wnt $/ \beta$-catenin- and Bmp-signaling pathways to mediate hair regeneration.

CXCL4 has been demonstrated to induce apoptosis of epithelia through activation of extrinsic and intrinsic death pathways $(16,26)$, which is regulated primarily by Bcl-2 family proteins. The Bcl-2 family of proteins are either anti-apoptotic
Od

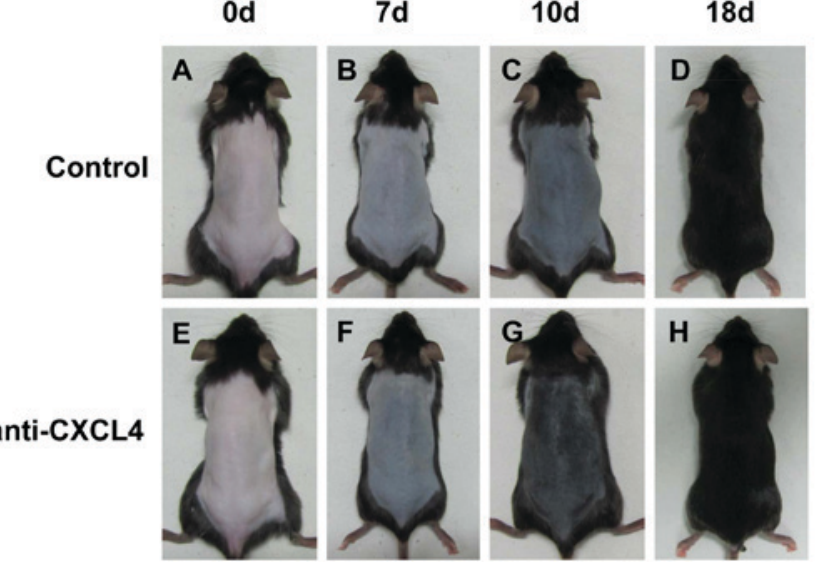

Figure 2. Anti-CXCL4 mAb promotes hair regeneration. Telogen-matched, 7-week-old C57BL/6 mice were depilated and subcutaneously injected with CXCL4 mAb $(n=10)$ or IgG as the control $(n=10)$ once weekly for 3 weeks. Photographic images were captured at $0,7,10$ and 18 days. Darker skin was observed in the CXCL4 mAb-treated mice compared with the control mice treated with isotype IgG. Control mice at (A) 0 days, (B) 7 days, (C) 10 days and (D) 18 days, and mice treated with CXCL4 antibody at (E) 0 days, (F) 7 days (G) 10 days and (H) 18 days. CXCL4, chemokine (C-X-C motif) ligand 4; $\mathrm{mAb}$, monoclonal antibody; $\mathrm{IgG}$, immunoglobulin $\mathrm{G}$.

or pro-apoptotic in nature, notably the anti-apoptotic Bcl-2 and pro-apoptotic Bax proteins. Bcl-2 is considered to protect cells from apoptosis, while Bcl-2 activity is determined by interaction with Bax (33). The present study demonstrated that CXCL4 mAb increased the expression of the anti-apoptotic $\mathrm{Bcl}-2$, whereas there was no significant change in the expression level of Bax in the skin at 10 days after depilation, at the anagen phase of hair follicles. Notably, the level of PCNA 

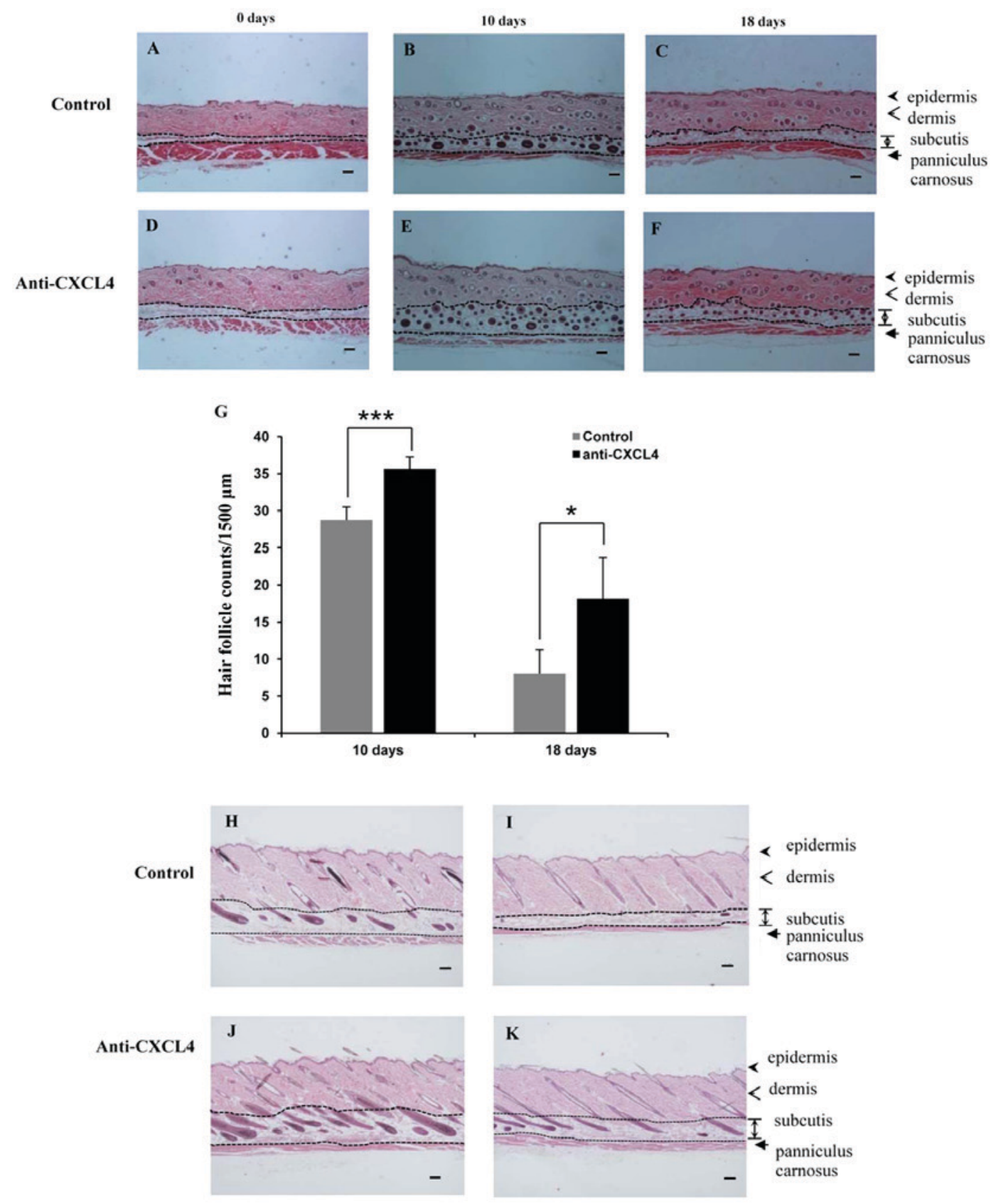

Figure 3. Hair follicles in the telogen-matched C57BL/6 mice treated with CXCL4 mAb. The effect of CXCL4 mAb on the hair follicles was analyzed using hematoxylin and eosin staining. (A-F) Transverse sections of the dorsal skin at 0,10 and 18 days were stained, and representative photomicrographs of skin sections are shown. Scale bar, $100 \mu \mathrm{m}$. Control mice at (A) 0 days, (B) 10 days and (C) 18 days, and mice treated with CXCL4 antibody at (D) 0 days, (E) 10 days and (F) 18 days. (G) The number of hair follicles in the deep subcutis in five fields per mouse was determined under a microscope (magnification, $\mathrm{x} 40$ ). Values are the mean \pm standard deviation ( $\mathrm{n}=5$ mice per group); $\mathrm{P}<0.05$ and ${ }^{* * *} \mathrm{P}<0.001$ as indicated. $(\mathrm{H}-\mathrm{K}$ ) Longitudinal sections of the dorsal skin were stained, and the images shown are representative of 5 mice. Scale bar, $100 \mu \mathrm{m}$. Control group at (H) 10 days and (I) 18 days and mice treated with CXCL4 $\mathrm{mAb}$ at $(\mathrm{J}) 10$ days and $(\mathrm{K}) 18$ days after depilation. CXCL4, chemokine (C-X-C motif) ligand 4; mAb, monoclonal antibody.

expression was clearly increased (Fig. 5). These results indicate that CXCL4 $\mathrm{mAb}$ promotes telogen to anagen transition by the stimulation of proliferation and suppression of apoptosis of hair follicular cells.

During hair follicle growth and hair production, factors promoting proliferation, differentiation and survival predominate in activity. However, hair follicle regression in the catagen phase is characterized by the activation of a number of signaling pathways that induce the apoptosis of hair follicle cells (34). In addition, in the process of hair follicle regression, caspase activation is required to induce apoptosis (35). Caspase-3 is a key executor of the apoptosis signal (36). In the present study, during the catagen phase (at 18 days after depilation), it was demonstrated that pro-apoptotic Bax expression was decreased, the expression of Bcl-2 was unchanged, and the expression of cleaved caspase- 3 was significantly decreased in the skin treated with CXCL4 mAb (Fig. 5). During the catagen phase, the dermal papilla transforms into a cluster of quiescent cells near to the regressing hair follicle epithelium, which moves from the subcutis to the border of the dermis and 
A

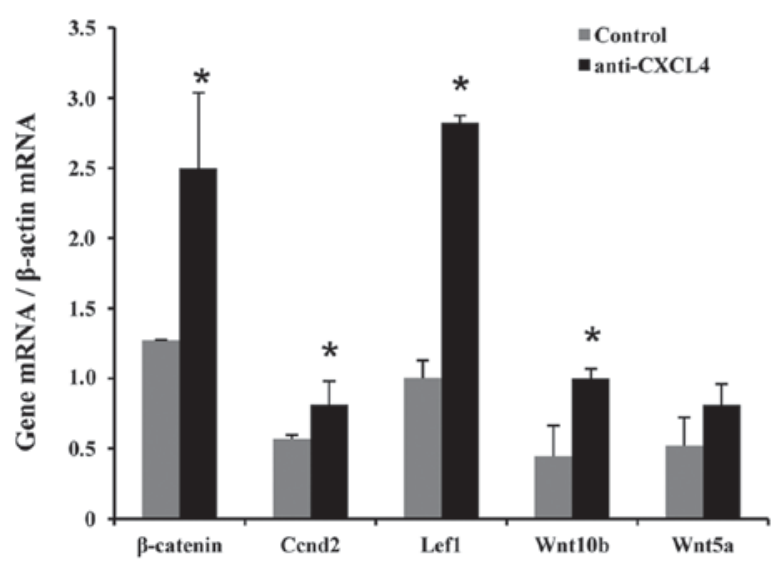

B

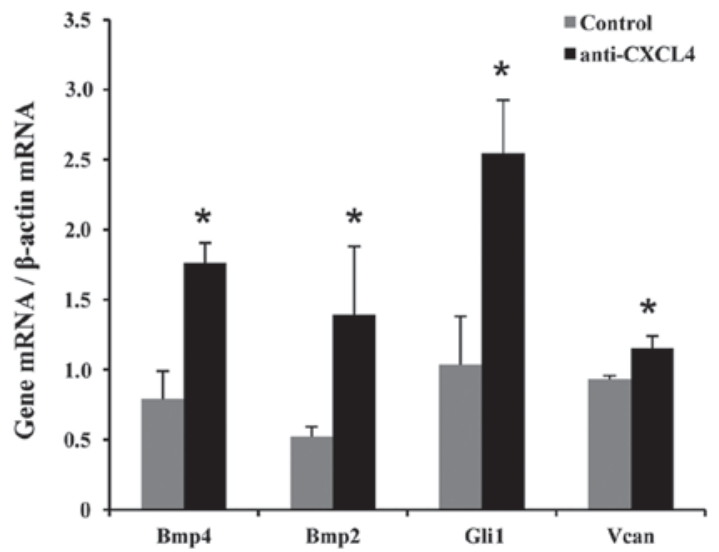

Figure 4. Wnt- and Bmp-signaling pathways are upregulated in the dorsal skin of mice at 10 days after CXCL4 mAb treatment. Relative mRNA expression levels of Wnt- (A) and Bmp- (B) signaling pathway genes were measured by reverse transcription-quantitative polymerase chain reaction with specific primers, with $\beta$-actin mRNA as the internal control. Data represent the mean \pm standard deviation for three independent experiments. $n=3$; ${ }^{P}<<0.05$ vs. the control group. CXCL4, chemokine (C-X-C motif) ligand 4; mAb, monoclonal antibody; Bmp, bone morphogenetic protein; Ccnd2, cyclin D2; Lef1, lymphoid enhancer binding factor 1; Gli1, GLI-Kruppel family member GLI1; Vcan, versican.

A

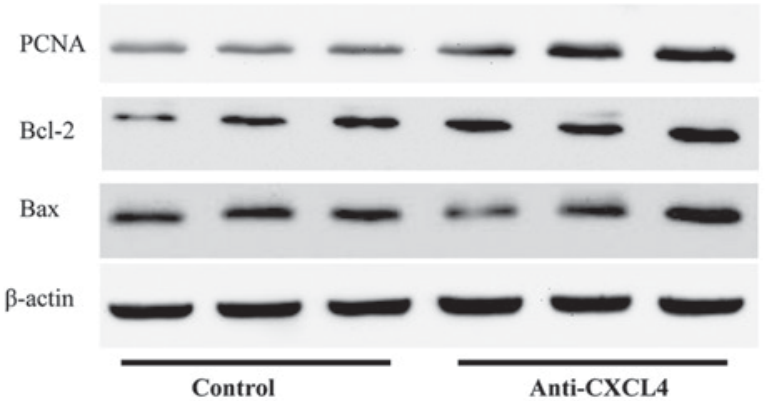

C

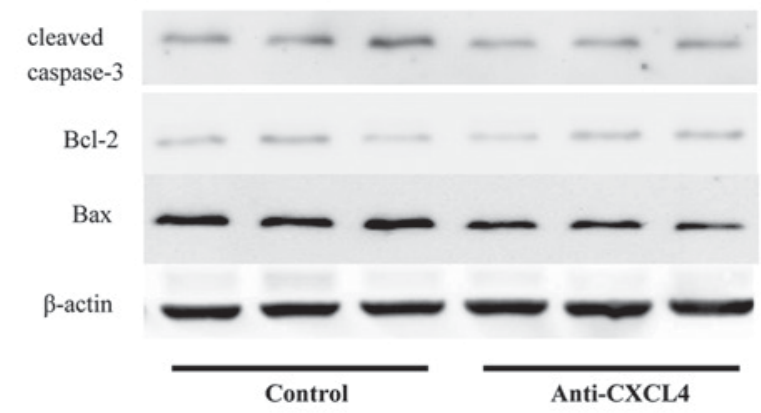

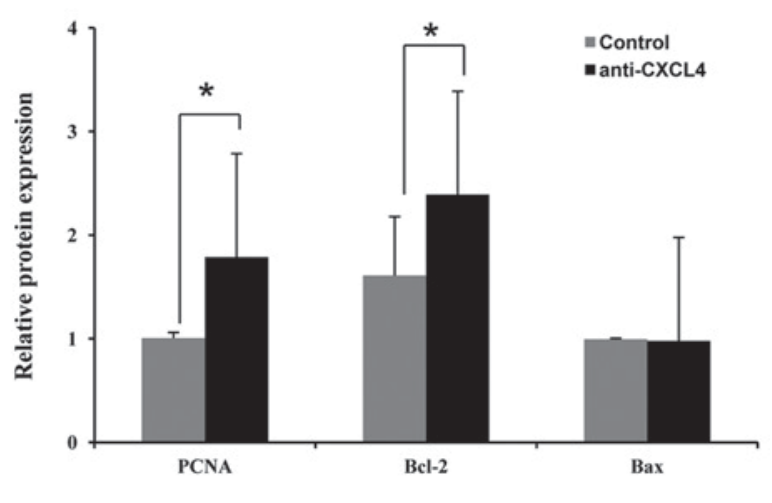

D

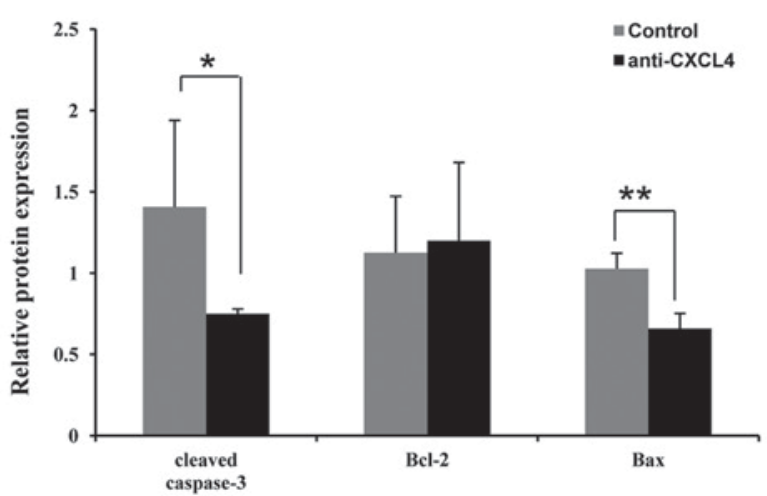

Figure 5. Effect of CXCL4 mAb on the expression of proliferation- and apoptosis-associated proteins. Depilated mice were treated with CXCL4 $\mathrm{mAb}$ or immunoglobulin $\mathrm{G}$ (control) once a week. Whole-tissue lysates of dorsal skin tissues at 10 and 18 days were subjected to western blot analysis. (A) Representative western blots and (B) quantification on day 10 and (C) representative western blots and (D) quantification on day 18. $\beta$-actin was used as the loading control. Data represent the mean \pm standard deviation $(n=3) .{ }^{*} \mathrm{P}<0.05$ and ${ }^{* *} \mathrm{P}<0.01$ as indicated. CXCL4, chemokine (C-X-C motif) ligand 4; mAb, monoclonal antibody; PCNA, proliferating cell nuclear antigen.

subcutis (35). In the present study, suppression of hair follicle apoptosis prevented the dermal papilla from being pulled upwards from the subcutis to the dermis in the catagen phase of hair follicles in the mice treated with CXCL4 mAb (Fig. 3). Thus, CXCL4 mAb attenuated the apoptosis of hair follicle cells in the catagen phase and delayed hair follicle regression.
In conclusion, blockade of CXCL4 using CXCL4 mAb accelerated hair growth through promotion of telogen to anagen transition, and delayed the regression of hair follicles in the catagen phase. In-depth studies are necessary to further elucidate the molecular mechanisms through which CXCL4 $\mathrm{mAb}$ imparts its hair growth activity. 


\section{Acknowledgements}

The present study was supported by the National Natural Science Foundation of China (No. 81273573) and the Science and Technology Commission of Shanghai Municipality (No. 11431921300).

\section{References}

1. Müller-Röver S, Handjiski B, van der Veen C, Eichmüller S, Foitzik K, McKay IA, Stenn KS and Paus R: A comprehensive guide for the accurate classification of murine hair follicles in distinct hair cycle stages. J Invest Dermatol 117: 3-15, 2001.

2. Schneider MR, Schmidt-Ullrich R and Paus R: The hair follicle as a dynamic miniorgan. Curr Biol 19: R132-R142, 2009.

3. Wang TL, Zhou C, Shen YW, Wang XY, Ding XL, Tian S, Liu Y, Peng GH, Xue SQ, Zhou JE, et al: Prevalence of androgenetic alopecia in China: A community-based study in six cities. Br J Dermatol 162: 843-847, 2010.

4. Cotsarelis G and Millar SE: Towards a molecular understanding of hair loss and its treatment. Trends Mol Med 7: 293-301, 2001.

5. Zhang NN, Park DK and Park HJ: Hair growth-promoting activity of hot water extract of Thuja orientalis. BMC Complement Altern Med 13: 9, 2013.

6. Miller MD and Krangel MS: Biology and biochemistry of the chemokines: A family of chemotactic and inflammatory cytokines. Crit Rev Immunol 12: 17-46, 1992.

7. Deutsch E, Johnson SA and Seegers WH: Differentiation of certain platelet factors related to blood coagulation. Circ Res 3 : $110-115,1955$

8. Rendu F and Brohard-Bohn B: The platelet release reaction: Granules' constituents, secretion and functions. Platelets 12 261-273, 2001.

9. Kasper B and Petersen F: Molecular pathways of platelet factor 4/CXCL4 signaling. Eur J Cell Biol 90: 521-526, 2011.

10. Scheuerer B, Ernst M, Dürrbaum-Landmann I, Fleischer J, Grage-Griebenow E, Brandt E, Flad HD and Petersen F: The CXC-chemokine platelet factor 4 promotes monocyte survival and induces monocyte differentiation into macrophages. Blood 95: 1158-1166, 2000

11. Han ZC, Lu M, Li J, Defard M, Boval B, Schlegel N and Caen JP: Platelet factor 4 and other CXC chemokines support the survival of normal hematopoietic cells and reduce the chemosensitivity of cells to cytotoxic agents. Blood 89: 2328-2335, 1997.

12. Gupta SK and Singh JP: Inhibition of endothelial cell proliferation by platelet factor- 4 involves a unique action on $\mathrm{S}$ phase progression. J Cell Biol 127: 1121-1127, 1994.

13. Luster AD, Greenberg SM and Leder P: The IP-10 chemokine binds to a specific cell surface heparan sulfate site shared with platelet factor 4 and inhibits endothelial cell proliferation. J Exp Med 182: 219-231, 1995.

14. Maione TE, Gray GS, Petro J, Hunt AJ, Donner AL, Bauer SI, Carson HF and Sharpe RJ: Inhibition of angiogenesis by recombinant human platelet factor- 4 and related peptides. Science 247 : 77-79, 1990.

15. Watson JB, Getzler SB and Mosher DF: Platelet factor 4 modulates the mitogenic activity of basic fibroblast growth factor. J Clin Invest 94: 261-268, 1994.

16. Gao J, Gao J, Qian L, Wang X, Wu M, Zhang Y, Ye H, Zhu S, Yu Y and Han W: Activation of p38-MAPK by CXCL4/CXCR3 axis contributes to p53-dependent intestinal apoptosis initiated by 5-fluorouracil. Cancer Biol Ther 15: 982-991, 2014.

17. Paus R, Stenn KS and Link RE: Telogen skin contains an inhibitor of hair growth. Br J Dermatol 122: 777-784, 1990.
18. Livak KJ and Schmittgen TD: Analysis of relative gene expression data using real-time quantitative PCR and the 2(-Delta Delta C(T)) Method. Methods 25: 402-408, 2001.

19. Plonka PM, Michalczyk D, Popik M, Handjiski B, Slominski A and Paus R: Splenic eumelanin differs from hair eumelanin in C57BL/6 mice. Acta Biochim Pol 52: 433-441, 2005.

20. Datta K, Singh AT, Mukherjee A, Bhat B, Ramesh B and Burman AC: Eclipta alba extract with potential for hair growth promoting activity. J Ethnopharmacol 124: 450-456, 2009.

21. Huelsken J, Vogel R, Erdmann B, Cotsarelis G and Birchmeier W: beta-Catenin controls hair follicle morphogenesis and stem cell differentiation in the skin. Cell 105: 533-545, 2001.

22. Van Mater D, Kolligs FT, Dlugosz AA and Fearon ER: Transient activation of beta-catenin signaling in cutaneous keratinocytes is sufficient to trigger the active growth phase of the hair cycle in mice. Genes Dev 17: 1219-1224, 2003.

23. Mundy G, Gutierrez G, Garrett R, Gallwitz W, Rossini G, Christiansen $\mathrm{C}$ and Langenberg A: Proteasome inhibitors stimulate both bone formation and hair growth by similar mechanisms. Ann N Y Acad Sci 1117: 298-301, 2007.

24. Dong L, Hao H, Xia L, Liu J, Ti D, Tong C, Hou Q, Han Q, Zhao Y, Liu H, et al: Treatment of MSCs with Wntla-conditioned medium activates DP cells and promotes hair follicle regrowth. Sci Rep 4: 5432, 2014.

25. Gentilini G, Kirschbaum NE, Augustine JA, Aster RH and Visentin GP: Inhibition of human umbilical vein endothelial cell proliferation by the CXC chemokine, platelet factor 4 (PF4), is associated with impaired downregulation of p21(Cip1/WAF1). Blood 93: 25-33, 1999

26. Woller G, Brandt E, Mittelstädt J, Rybakowski C and Petersen F: Platelet factor 4/CXCL4-stimulated human monocytes induce apoptosis in endothelial cells by the release of oxygen radicals. J Leukoc Biol 83: 936-945, 2008.

27. Soma T, Fujiwara S, Shirakata Y, Hashimoto K and Kishimoto J: Hair-inducing ability of human dermal papilla cells cultured under Wnt $/ \beta$-catenin signalling activation. Exp Dermatol 21: 307-309, 2012.

28. Fuchs E and Raghavan S: Getting under the skin of epidermal morphogenesis. Nat Rev Genet 3: 199-209, 2002.

29. Li YH, Zhang K, Yang K, Ye JX, Xing YZ, Guo HY, Deng F, Lian XH and Yang T: Adenovirus-mediated Wnt10b overexpression induces hair follicle regeneration. J Invest Dermatol 133: 42-48, 2013.

30. Andl T, Reddy ST, Gaddapara T and Millar SE: WNT signals are required for the initiation of hair follicle development. Dev Cell 2: 643-653, 2002.

31. Gat U, DasGupta R, Degenstein L and Fuchs E: De Novo hair follicle morphogenesis and hair tumors in mice expressing a truncated beta-catenin in skin. Cell 95: 605-614, 1998.

32. Jiang S, Zhao L, Teklemariam T and Hantash BM: Small cutaneous wounds induce telogen to anagen transition of murine hair follicle stem cells. J Dermatol Sci 60: 143-150, 2010.

33. Adams JM and Cory S: The Bcl-2 protein family: Arbiters of cell survival. Science 281: 1322-1326, 1998.

34. Lindner G, Botchkarev VA, Botchkareva NV, Ling G, van der Veen $\mathrm{C}$ and Paus R: Analysis of apoptosis during hair follicle regression (catagen). Am J Pathol 151: 1601-1617, 1997.

35. Botchkareva NV, Ahluwalia G and Shander D: Apoptosis in the hair follicle. J Invest Dermatol 126: 258-264, 2006.

36. Sabbagh L, Kaech SM, Bourbonnière M, Woo M, Cohen LY, Haddad EK, Labrecque N, Ahmed R and Sékaly RP: The selective increase in caspase- 3 expression in effector but not memory $\mathrm{T}$ cells allows susceptibility to apoptosis. J Immunol 173: 5425-5433, 2004. 